\title{
Improved R-wave detection for enhanced cardiac Gating using an MRI-compatible 12-lead ECG and multi-channel analysis
}

Zion Tsz Ho Tse ${ }^{1 *}$, Charles L Dumoulin², Gari Clifford ${ }^{3}$, Michael Jerosch-Herold ${ }^{1}$, Daniel Kacher ${ }^{1}$, Raymond Kwong ${ }^{1}$, William Gregory Stevenson ${ }^{1}$, Ehud Jeruham Schmidt ${ }^{1}$

From 2011 SCMR/Euro CMR Joint Scientific Sessions

Nice, France. 3-6 February 2011

\section{Background}

An important requirement for successful cardiac MRI is accurate gating. However, obtaining proper Electrocardiogram (ECG) gating inside the MRI is a difficult problem, due to the Magneto-Hydro-Dynamic (MHD) effect, resulting in frequent intermittent gating. The MHD effect generates a voltage due to conductive blood flow perpendicular to $\mathrm{B}_{0}$, and distorts the real ECG. Intermittent gating also occurs when arrhythmia patients incur several events of arrhythmia between their sinus rhythm (SR) beats, resulting in blurred images. We hypothesized that a novel real-time 3-D gating method could accurately detect the QRS complex, even in difficult cases, such as with Premature Ventricular Contractions (PVCS), non-stable heart rate (atrial fibrillation, exercise), imaged at both 1.5 and 3T MRIs.

\section{Methods}

Fig. 1 shows our ECG system [1]. A gating method based on a 3-D ECG representation is developed.

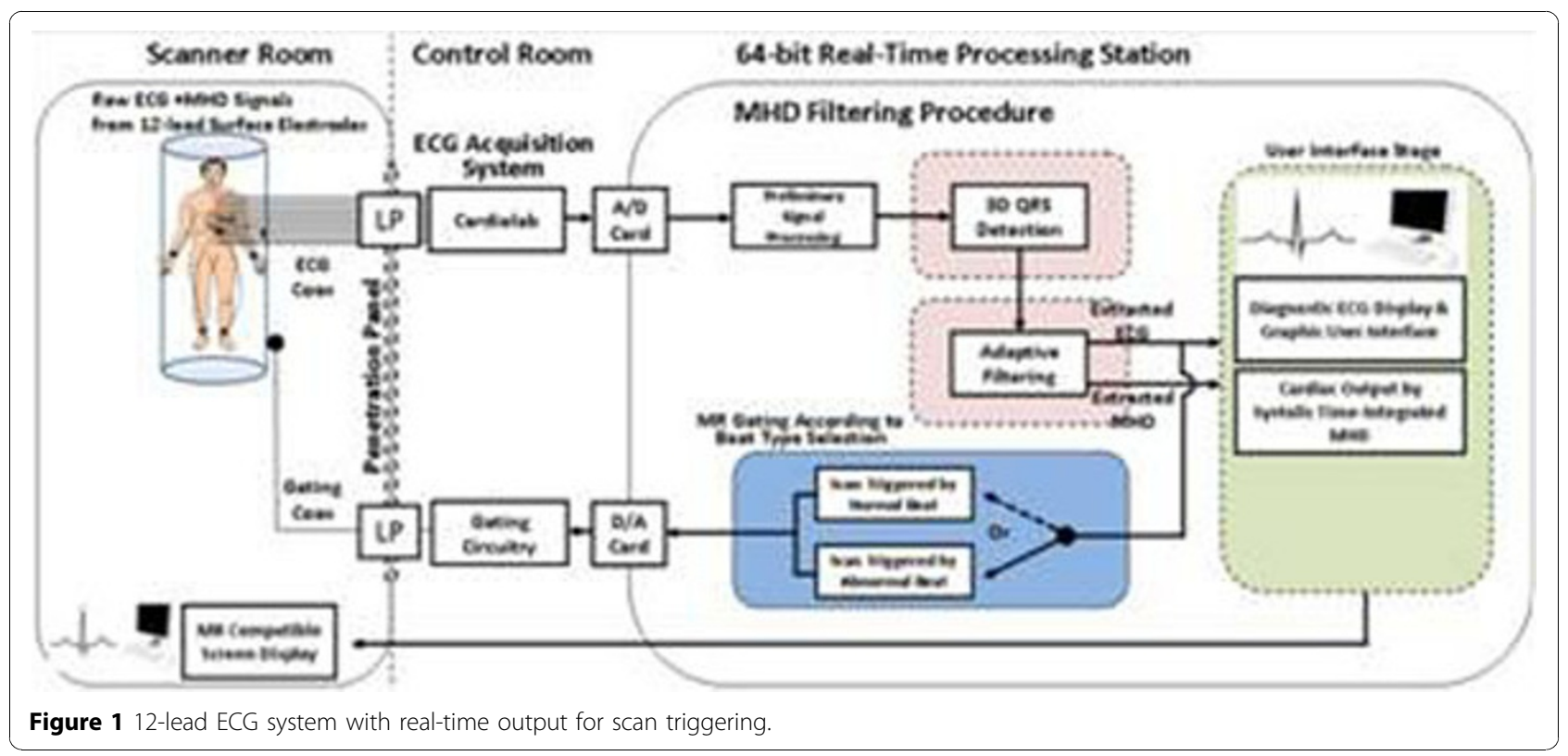

'Brigham and Women's Hospital, Boston, MA, USA

Full list of author information is available at the end of the article

BioMed Central $\begin{aligned} & \text { O } 2011 \text { Tse et al; licensee BioMed Central Ltd. This is an open access article distributed under the terms of the Creative Commons } \\ & \text { any medium, provided the original work is properly cited. }\end{aligned}$
any), which permits unrestricted use, distribution, and reproduction in 


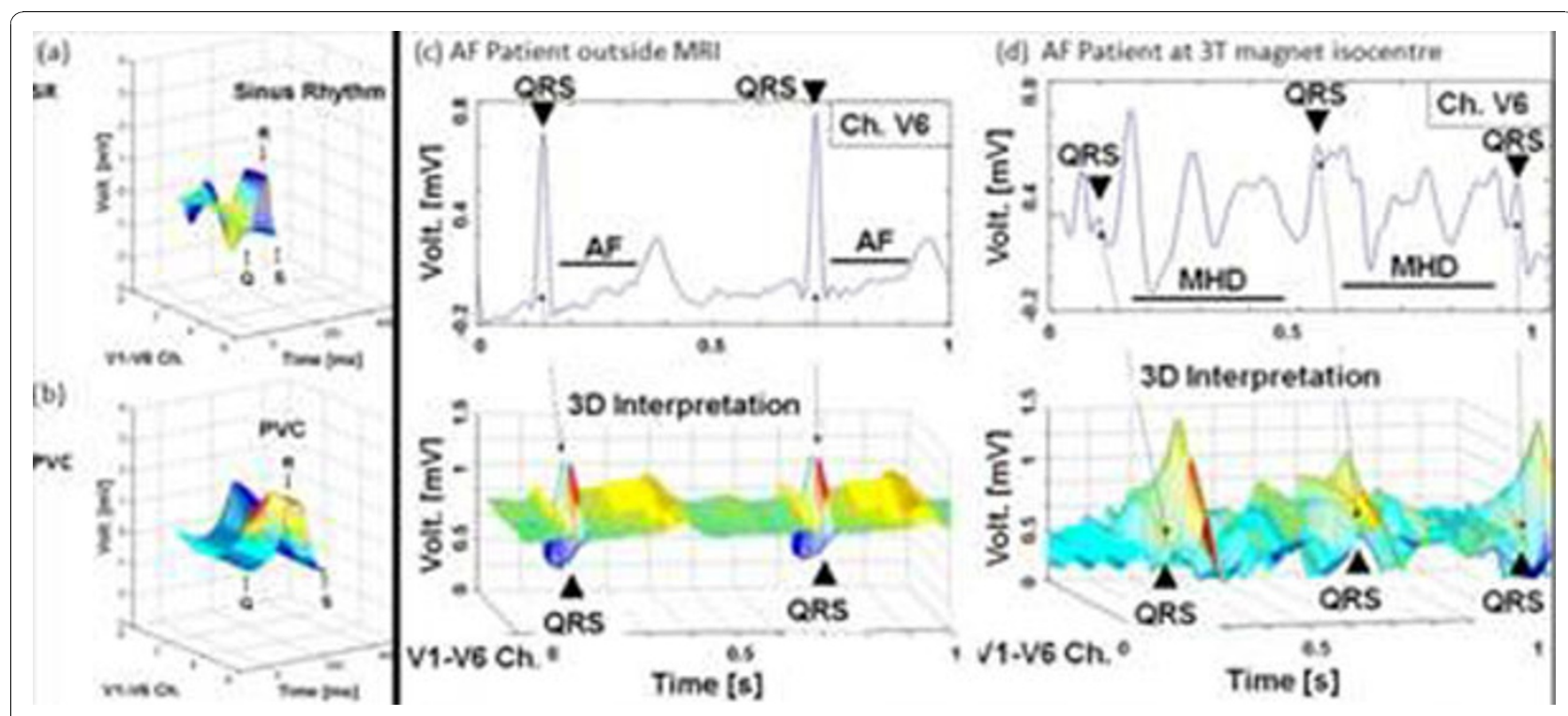

Figure 2 3-D representation of ECG signals: PVS pateint's (a) and SR (b) PVC beats taken outside a 3T. AF patient's ECG (c) outside and (d) inside a 3T: Channel V6 signal (top) and 3D representation (bottom). 3D-QRS is identifiable even with stronger MHD peak.

By making ECG channels V1-V6 represent a third axis, an additional dimension is added to the time and voltage axes. The channel axis (Fig. 2(a-b)) carries extra information on the electrical signal propagating from the source in the heart to the surface leads. Within this, the QRS complex forms a unique 3-D geometry, which is identifiable even with a large MHD effect using a fast
FFT 2-D cross-correlation subroutine to achieve a realtime computation speed of $<5 \mathrm{msec}$.

\section{Results}

Fig. 2(a-b) shows the 3-D QRS kernels of SR and PVC beats. The PVC kernel (b) is significantly different from the SR (a) not only along the time axis but also the

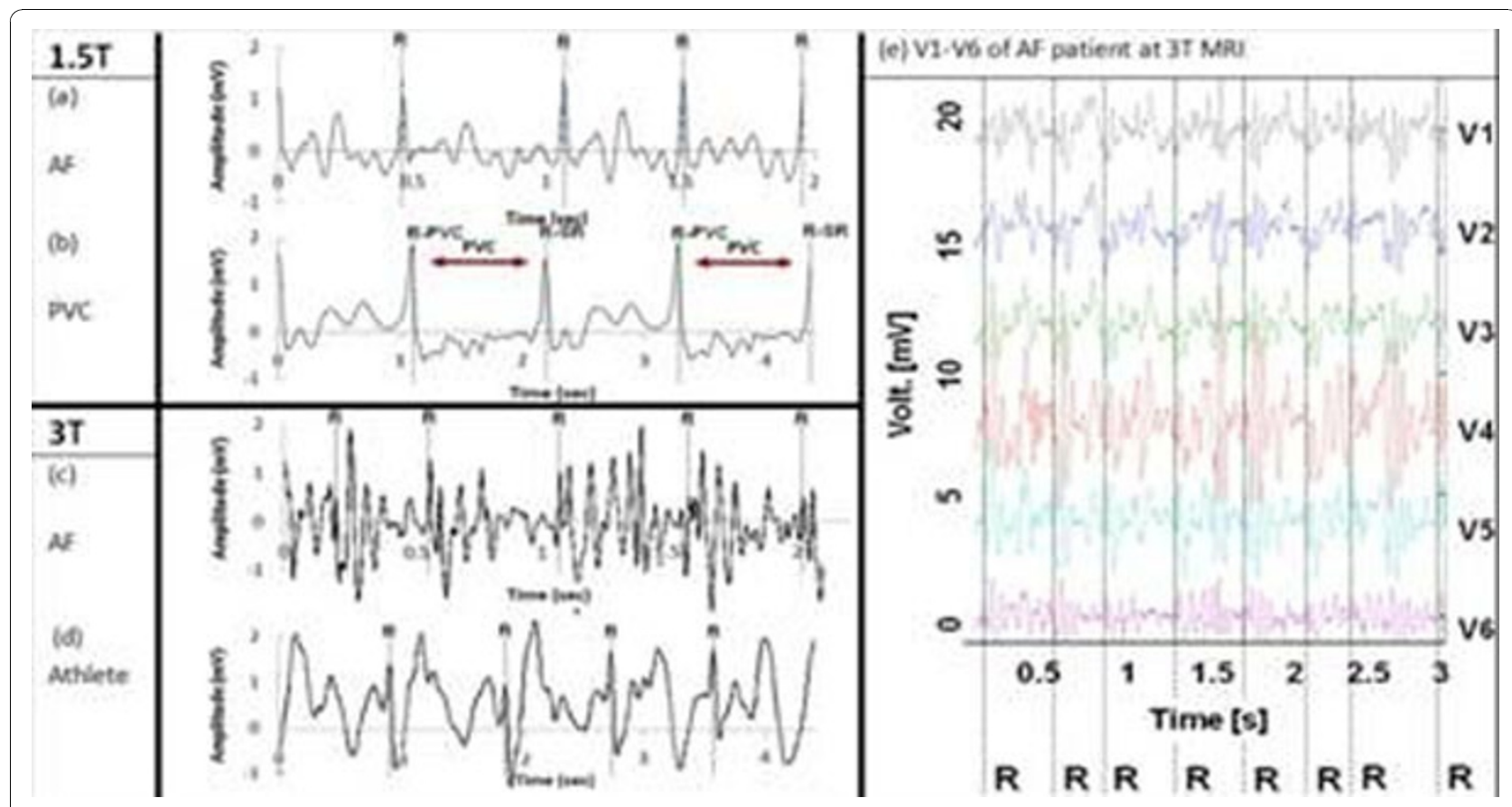

Figure 3 (a-d) 20-sec breath-held ECGs obtained at 1.5T (a,b) and 3T (c,d) MRIs for AF, PVC and exercising athlete subjects. R designates detected R-wave peaks using 3D-QRS. (e) V1-V6 traces of the AF patient at 3T. All R peaks (dotted lines) are correctly identified (a-e). 
channel axis. Because PVC foci are located at ventricular locations, source location and electrical wave propagation to the surface electrodes are different, which affect the channels axis, providing a unique 3-D geometry distinguishable from the SR kernel. Fig. 2(d) shows ECGs of an Atrial Fibrillation (AF) patient taken inside a 3T, in which the QRS complex is hardly identified in V6 (top), smaller than the MHD signal. However, the SR QRS kernels (bottom) are distinguishable with their unique 3-D features in Fig. 2(c-d), since the MHD originates from the aortic arch and SR from sinus node. Fig. 3 shows the gating results of $2 \mathrm{AF}$ and $1 \mathrm{PVC}$ patient, as well as 1 athlete subject exercising to produce heart rate variation from $44 \mathrm{bpm}$ to $87 \mathrm{bpm}$.

\section{Conclusions}

The gating method, based on a unique 3-D ECG representation, allows accurate R-wave detection and separation of beat types even with strong MHD signals.

\section{Author details}

'Brigham and Women's Hospital, Boston, MA, USA. ${ }^{2}$ University of Cincinnat College of Medicine, Cincinnati, OH, USA. ${ }^{3}$ University of Oxford, Oxford, UK.

Published: 2 February 2011

\section{Reference}

1. Tse : ISMRM 2010

doi:10.1186/1532-429X-13-S1-P3

Cite this article as: Tse et al:: Improved R-wave detection for enhanced cardiac Gating using an MRI-compatible 12-lead ECG and multi-channel analysis. Journal of Cardiovascular Magnetic Resonance 2011 13(Suppl 1):P3.
Submit your next manuscript to BioMed Central and take full advantage of:

- Convenient online submission

- Thorough peer review

- No space constraints or color figure charges

- Immediate publication on acceptance

- Inclusion in PubMed, CAS, Scopus and Google Scholar

- Research which is freely available for redistribution

Submit your manuscript at www.biomedcentral.com/submit 